\section{Prevalência de pressão arterial elevada em crianças e adolescentes: revisão sistemática}

Diego Giulliano Destro Christofaro 1

Selma Maffei de Andrade 2

Rômulo Araújo Fernandes 3

Marcos Aparecido Sarria Cabrera 4

Raphael Mendes Ritti-Dias 5

\section{The prevalence of high arterial blood pressure in children and adolescents: a systematic review}

\section{Abstract}

Objectives:to identify the prevalence of high blood pressure in children and adolescents in studies published in the past 30 years.

Methods: a search was carried out in the Medline/PubMed, Lilacs, Web of Science, Embase and Scielo databases for articles published between 1980 and 2009. Articles were included if they were original studies of the prevalence of high arterial blood pressure or hypertension in a sample of more than 500 subjects.

Results: the prevalence of high blood pressure (in individuals aged between 4 and 20 years) varied from $0.46 \%$ to $20.6 \%$. The lower prevalence of high blood pressure was found in studies where the majority of the sample was aged between 4 and 7 years. The protocols most commonly used for evaluation of blood pressure were those developed by the Task Force and National High Blood Pressure Education Program. In all, $52.4 \%$ of studies used auscultation to determine blood pressure and $47.6 \%$ used oscillometry.

Conclusions: despite the great discrepancy between the values for prevalence found in the studies, it is clear that there has been a growth in the prevalence of high blood pressure in children and adolescents all over the world.

Key words Prevalence, High blood pressure, Child, Adolescent

\section{Resumo}

Objetivos: identificar as prevalências de pressão arterial elevada em crianças e adolescentes em estudos publicados nos últimos 30 anos.

Métodos: foi realizada uma busca nas seguintes bases de dados: Medline/PubMed, Lilacs, Web of Science, Embase e Scielo entre os anos de 1980 e 2009. Foram incluidos estudos originais de prevalência de pressão arterial elevada ou hipertensão com tamanho amostral superior a 500 sujeitos.

Resultados: a prevalência de pressão arterial elevada em crianças e adolescentes de 4 a 20 anos variou de 0,46\% a 20,6\%. As menores prevalencias de pressão arterial elevada foram detectadas nos estudos em que a maior parte da amostra foi composta por crianças de 4 a 7 anos. Os protocolos mais utilizados na avaliação da pressão arterial foram o da Task Force e o do National High Blood Pressure Education Program. Ao todo 52,4\% dos estudos utilizaram o método auscultatório para verificar a medida de pressão arterial e 47,6\%, o método oscilométrico.

Conclusões: apesar da grande discrepância dos valores de prevalência encontrados, ressalta-se o crescimento da prevalência de pressão arterial elevada na população de crianças e adolescentes de diversas partes do mundo.

Palavras-chave Prevalência, Pressão arterial elevada, Criança, Adolescente 


\section{Introdução}

Ao longo das últimas décadas, tem sido observado aumento substancial no número de óbitos decorrentes de agravos e doenças não transmissíveis em diversos países. Entre essas doenças, destacam-se as cardiovasculares (DCV), que são, atualmente, as principais causas de óbito responsáveis por aproximadamente $40 \%$ da mortalidade mundial. ${ }^{1}$

A hipertensão arterial é considerada o principal fator de risco cardiovascular, uma vez que potencializa a ocorrência de diversas DCV (insuficiência cardíaca, insuficiência renal, doença arterial periférica, entre outras), de forma que seu diagnóstico precoce vem sendo enfatizado como importante estratégia de saúde pública. Diversos estudos têm mostrado altas prevalências de pressão arterial elevada (PAE) em crianças e adolescentes de diferentes países. $2-5$ No entanto, essas prevalências parecem variar de acordo com uma série de fatores, tais como a região, o sexo, a faixa etária e o local de residência.

Ainda que os fatores genéticos e ambientais sejam determinantes na ocorrência da hipertensão arterial, observa-se que os estudos rea-lizados em diferentes regiões do mundo variam quanto aos procedimentos metodológicos empregados, que incluem desde a faixa etária da amostra até os protocolos de coleta e os aparelhos para a medida da pressão arterial. Assim, é possível que a variabilidade dos resultados observados entre os estudos possam ter sido decorrentes dessas diferenças. Portanto, a síntese dos resultados de estudos sobre a prevalência de PAE em crianças e adolescentes pode auxiliar na interpretação desses achados à luz dos procedimentos metodológicos empregados. Além disso, pela presente revisão, será possível verificar se houve ou não variação entre as prevalências de PAE observadas pelos diferentes estudos.

O objetivo do presente estudo foi descrever a prevalência de PAE de acordo com as características da amostra e os procedimentos metodológicos empregados.

\section{Métodos}

A revisão da literatura de artigos publicados de 1980 até 2009 foi realizada durante os meses de setembro de 2009 a agosto de 2010 nas bases de dados eletrônicas Medline/PubMed, Lilacs, Web of Science, Embase e Scielo. Os seguintes descritores/termos foram utilizados na pesquisa: hipertensão arterial, pressão arterial elevada, crianças, adolescentes, prevalência. Em inglês foram empregados os seguintes descritores/termos: hypertension, high blood pressure, elevated blood pressure, children, teenager, adolescents, prevalence.

Inicialmente, foi feita a leitura dos títulos dos estudos para identificação daqueles que se enquadrariam dentro dos critérios estabelecidos para a revisão. $\mathrm{Na}$ impossibilidade da tomada de decisão com a leitura do título, foi feita a leitura do resumo. Para aqueles estudos que, mesmo com a leitura e do título e do resumo, ainda não tenha sido possível determinar se atenderiam aos critérios de inclusão, foi feita a leitura do manuscrito na íntegra.

Como critérios de inclusão, os estudos deveriam: a) ter sido realizados com crianças e/ou adolescentes, sem delimitação da idade mínima, porém com idade máxima de 20 anos. Apesar do período de adolescência compreender a faixa etária dos 10 aos 19 anos, um dos estudos incluiu escolares com idade de 20 anos, por apresentar majoritariamente dados sobre adolescentes, foi mantido na presente revisão; b) apresentar amostra igual ou superior a 500 sujeitos; e c) apresentar dados originais de prevalência de hipertensão ou PAE. Artigos que não informassem o instrumento utilizado para medir a pressão arterial, faixa etária ou que fossem de revisão foram excluídos do estudo. Ao todo foram encontrados 743 artigos com os descritores utilizados. Destes, 683 foram excluídos a partir da leitura do título ou do resumo. Restaram 40 estudos para leitura do texto na íntegra, dos quais 21 avaliaram as taxas de prevalência.

Os artigos incluídos no estudo foram caracterizados quanto: a) país em que foi desenvolvido; b) ano de publicação; c) periódico de publicação; d) tamanho da amostra; e) faixa etária da amostra; f) método utilizado para medida da pressão arterial; g) número de aferições realizadas; h) terminologia utilizada; i) critério utilizado; j) prevalência identificada; k) protocolo empregado.

\section{Resultados}

Na Tabela 1, são apresentadas as características dos estudos incluídos nesta revisão. Dos 21 estudos analisados, 19 (90,4\%) foram realizados em escolas e, apenas dois, nas comunidades $(9,6 \%)$. Doze desses estudos foram realizados no continente Americano $(57,1 \%)$, quatro na Europa $(19,1 \%)$, três no continente Asiático $(14,3 \%)$ e dois na África $(9,7 \%)$. Cinco estudos $(23,8 \%)$ tiveram o seu tamanho amostral variando entre 500 e 1000 sujeitos, em 10 $(47,6 \%)$ o tamanho amostral variou de 1001 a 5000 pessoas, em quatro $(19,1 \%)$ foi encontrado amostra entre 5001 e 10.000 e em dois estudos $(9,5 \%)$ a 
amostra avaliada foi superior a 10.000 sujeitos. As idades estudadas variaram de 4 a 20 anos. A maioria dos estudos, 85,7\% $(\mathrm{n}=18)$ considerou 17 anos como idade limite, e em apenas três $(14,7 \%)$ a idade limite foi acima dos 17 anos. A maior variação entre a idade mínima e máxima foi observada em um estudo $(4,7 \%)$, que apontou uma diferença de 13 anos, enquanto a menor discrepância observada entre as faixas etárias foi de três anos, relatadas em dois estudos $(9,5 \%)$.

Na Tabela 2 estão apresentadas a prevalência de PAE e as características metodológicas dos estudos incluídos.

Houve grande variação na prevalência de PAE entre os estudos. $\mathrm{O}$ estudo que apresentou a menor prevalência foi realizado na Índia por Anand et al., 6 onde foi encontrada prevalência de $0,46 \%$ de PAE. $\mathrm{O}$ estudo de Urrutia-Rojas et al.,4 realizado no Estado do Texas, Estados Unidos, foi o que encontrou a maior prevalência de PAE $(20,6 \%)$ geral (sem estratificar por idade e sexo). A maioria dos estudos $(66,7 \%)$ observou prevalência de PAE variando de $3 \%$ a $10 \%$. Houve proporções similares, nos estudos, quanto à utilização dos métodos auscultatório e oscilométrico para medidas da pressão arterial: $52,4 \%$ para o método auscultatório e $47,6 \%$ para o oscilométrico. Uma parte dos estudos $8,12,18,20,24$ mediu a pressão arterial em dias diferentes. $\mathrm{O}$ número de aferições da pressão arterial mais realizadas em um único dia foi três vezes. Além disso, os estudos que realizaram medidas consecutivas da pressão arterial em um único dia e que mostraram a prevalência de PAE a cada medida observaram que a prevalência de PAE tende a diminuir conforme o aumento do número de medidas. ${ }^{3,16}$

Houve, no conjunto, valores semelhantes quanto à utilização dos protocolos da Task Force (versão de 1987 e 1996)25,26 e do National High Blood Pressure Education Program (NHBPEP; 2004)27 (que é uma atualização dos critérios da Task Force): dos 21 estudos analisados, dez utilizaram os critérios do NHBPEP $(47,6 \%)$ e nove o da Task Force $(42,8 \%)$. Apenas dois estudos não utilizaram os critérios da Task Force e do NHBPEP $(9,6 \%)$. O critério mais utilizado para detecção da PAE foram valores situados acima do percentil 95, levando-se em consideração o sexo, a idade e a estatura dos indivíduos avaliados.

Tabela 1

Características gerais dos estudos sobre prevalência de pressão arterial elevada em crianças e adolescentes.

\begin{tabular}{|c|c|c|c|c|c|c|}
\hline Estudo & País & Ano & Periódico & Local de coleta & $\begin{array}{l}\text { Tamanho } \\
\text { amostral }\end{array}$ & $\begin{array}{c}\text { Faixa etária } \\
\text { (anos) }\end{array}$ \\
\hline Anand et al. 6 & Índia & 1996 & Indian Pediatr & Escolares & 5000 & $5-17$ \\
\hline Oliveira et al. 7 & Brasil & 2004 & Arq Bras Endocrinol & Escolares & 701 & $5-9$ \\
\hline Sánches-Zamorano et al.8 & México & 2009 & Prev Medicine & Escolares & 2387 & $12-15$ \\
\hline Ostrowska-Nawarycz et al.9 & Polônia & 2007 & Kardiol Pol & Escolares & 25.309 & 7-19 \\
\hline Monyeki et al.10 & África do Sul & 2006 & Int J Epidemiol & Escolares & 1884 & $6-13$ \\
\hline Monego et al.11 & Brasil & 2006 & Arq Bras Cardiol & Escolares & 3169 & $7-14$ \\
\hline Saleh et al.12 & Kuwait & 2000 & East Mediterr Health J & Escolares & 1312 & $6-10$ \\
\hline Salvadori et al.13 & Canadá & 2008 & Pediatrics & Comunidade & 675 & 4-17 \\
\hline Silva e Farias Jr. 14 & Brasil & 2004 & Rev Bras Med Esporte & Escolares & 674 & $14-17$ \\
\hline Mavrakanas et al.15 & Grécia & 2009 & Rural and Remote Health & Escolares & 572 & 4-10 \\
\hline Borges et al. 16 & Brasil & 2007 & Rev Saúde Publica & Escolares & 601 & 7-10 \\
\hline Genovesi et al.17 & Itália & 2005 & J Hypertens & Escolares & 2416 & $6-11$ \\
\hline Moura et al. 3 & Brasil & 2004 & J Pediatr (Rio J) & Escolares & 1253 & 7-17 \\
\hline McNiece et al. 18 & Estados Unidos & 2007 & J Pediatr & Escolares & 6790 & $11-17$ \\
\hline Chiolero et al. 19 & Rep. de Seychelles & 2007 & J Hum Hypertension & Escolares & 15.612 & 5-16 \\
\hline Chiolero et al.20 & Suiça & 2007 & J Pediatric & Escolares & 5207 & $11-17$ \\
\hline Jafar et al.21 & Pakistão & 2005 & Circulation & Comunidade & 5461 & $5-14$ \\
\hline Gomes e Alves 22 & Brasil & 2009 & Cad Saude Publica & Escolares & 1878 & $14-20$ \\
\hline Paradis et al.23 & Canadá & 2004 & Circulation & Escolares & 3589 & 9-16 \\
\hline Sorof et al.24 & Estados Unidos & 2004 & Pediatrics & Escolares & 5102 & $10-19$ \\
\hline Urrutia Rojas et al.4 & Estados Unidos & 2006 & BMC Pediatrics & Escolares & 1066 & 8-13 \\
\hline
\end{tabular}




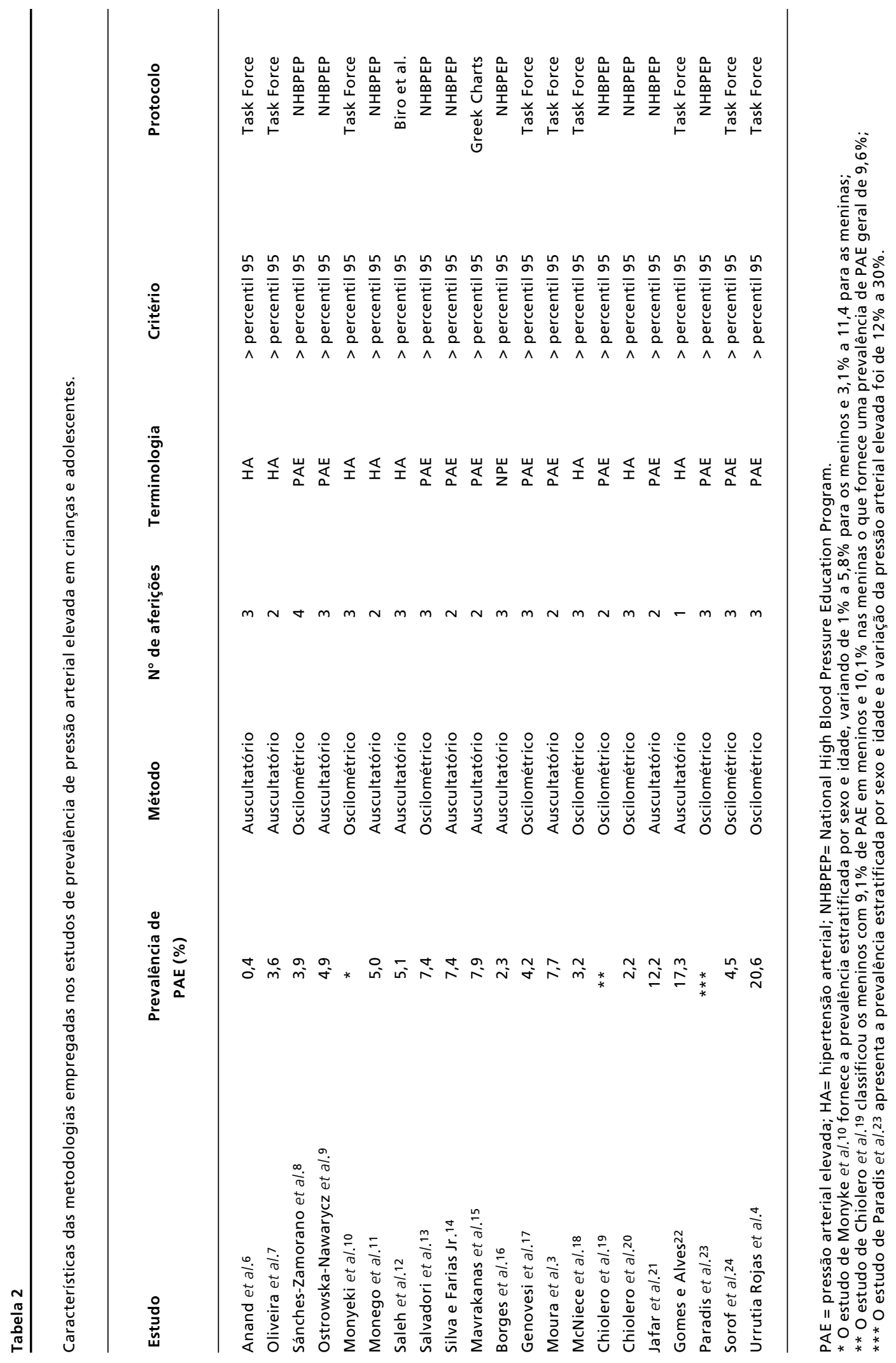




\section{Discussão}

Os dados sintetizados nessa revisão trazem algumas informações relevantes: a) há grande variação na prevalência de PAE entre crianças e adolescentes nos estudos; b) a maioria dos estudos sobre esta temática foi realizada na última década; c) observaram-se diferenças nos métodos utilizados e no número de aferições para a medida da pressão arterial; d) a nomenclatura utilizada foi distinta entre os estudos; e) em geral, há concordância quanto aos critérios de classificação utilizados para detectar a PAE.

As prevalências encontradas em diversas regiões do mundo nos estudos analisados variaram de $0,46 \%$, na Índia, entre crianças e adolescentes de 517 anos, 6 a 20,6\% nos Estados Unidos, na faixa etária de 8-13 anos. ${ }^{4}$ Essa variação pode ter sido acarretada por diferenças metodológicas entre os estudos, tais como os pontos de corte utilizados ou a idade da amostra. Ressalta-se ainda que no estudo de Anand et al. 6 a prevalência de excesso de peso na Índia foi de aproximadamente $3,5 \%$ enquanto no estudo americano 4 a prevalência de obesidade foi de $23,5 \%$ para adolescentes caucasianos, 31,9\% para adolescentes hispânicos e 32,8\% para afro-americanos. Considerando que a obesidade é um dos principais fatores de risco para a hipertensão em crianças e adolescentes, é possível que esta característica também tenha contribuído para a variada prevalência de PAE entre os estudos.

A maioria dos estudos analisados foi publicada a partir do ano 2000, revelando o recente e crescente interesse em pesquisas sobre a PAE em crianças e adolescentes. Observou-se número praticamente equivalente dos métodos utilizados para a mensuração da pressão arterial: auscultatório com utilização da coluna de mercúrio ou aneróide, e o método oscilométrico por meio de aparelhos digitais.

A utilização do método auscultatório pode apresentar limitações em estudos epidemiológicos, uma vez que a precisão nas medidas da pressão arterial obtida é dependente do avaliador e, nesses estudos, geralmente há necessidade de mais de um avaliador. Uma alternativa para a avaliação da medida da pressão arterial em estudos epidemiológicos tem sido a utilização dos aparelhos oscilométricos. $5,28-30$ Entretanto, como alguns desses aparelhos podem superestimar ou subestimar os valores de pressão arterial,31 é importante que sejam utilizados aparelhos validados e devidamente calibrados. No caso de adolescentes, já existem equipamentos validados para esse fim. ${ }^{32,33}$ No entanto, é importante considerar alguns cuidados para a utilização de tais equipamentos na prática. Por exemplo, como com qualquer aparelho eletrônico, é necessário manter constante atenção ao estado das pilhas, pois carga baixa dessa fonte de energia pode gerar erros nos dados obtidos. Além disso, o aparelho pode apresentar erros de medida em crianças (geralmente com idade inferior a nove anos) ou adolescentes com a circunferência de braço muito pequena, sendo então necessária a utilização do método auscultatório.

Diversos protocolos têm sido empregados para a detecção dos valores de PAE em crianças e adolescentes. Os mais utilizados têm sido o da Task Force (1987 e 1996)25,26 e o do National High Blood Pressure Education Program (2004).27 Apenas dois estudos relataram utilizar diferentes tabelas normativas para a classificação da PAE. ${ }^{34,35}$ Os protocolos da Task Force 25,26 e do National High Blood Pressure Education Program 27 foram elaborados com amostra de crianças e adolescentes americanos, país com elevada prevalência de PAE, o que pode influenciar as comparações com outros pontos de corte para PAE, quando desenvolvidos em países com menor taxa deste desfecho. Diferenças entre prevalências de acordo com os pontos de corte adotados também foram encontrados por Moraes et al. 36 ao avaliarem 19 estudos sobre prevalência de síndrome metabólica em adolescentes, variável na qual um dos componentes é a hipertensão arterial.

Por meio da presente revisão foi possível identificar que os termos utilizados para denominar valores elevados de pressão arterial foram diferentes entre os estudos, pois enquanto alguns estudos utilizaram o termo hipertensão arterial, outros estudos utilizaram o termo PAE. A denominação hipertensão arterial parece ser correta apenas nos casos em que a avaliação das medidas tenha ocorrido em dias diferentes, seguindo os procedimentos descritos para o diagnóstico da doença. ${ }^{27}$ Por outro lado, o termo PAE parece ser mais apropriado quando a pressão arterial é avaliada em apenas uma ocasião.

Em poucos dos estudos analisados houve menção ao horário em que foram realizadas as coletas das medidas de pressão arterial. Este fato é importante, visto que a medida da pressão arterial no período matutino é mais propensa a detectar valores mais baixos, próximos dos níveis basais. ${ }^{5}$ Reconhece-se, porém, a dificuldade para padronização do horário da medida de pressão arterial em estudos epidemiológicos, além do que a maioria dos estudos realizados com crianças e adolescentes ocorreu em escolas, onde, frequentemente, o avaliador necessita se ajustar aos horários de funcionamento para realizar as coletas.

A grande variação das faixas etárias estudadas (4 
a 20 anos) também contribuiu para a ampla variação das prevalências. No estudo realizado por Anand et al., ${ }^{6}$ na Índia, a prevalência de PAE encontrada $(0,46 \%)$ pode ser considerada baixa. Entretanto, na amostra analisada por esse estudo constata-se que o número de sujeitos com idade entre cinco e dez anos era superior ao da faixa etária de 11-17 anos. Como a prevalência de PAE tende a aumentar com a idade, a quantidade de indivíduos nas diferentes faixas etárias também é outro fator limitante na comparação dos estudos. Ressalta-se ainda que a maioria dos estudos não fez a estratificação da amostra separando crianças e adolescentes, o que limitou a descrição da prevalência de PAE por faixa etária.

Outro fator que parece influenciar as estimativas de PAE em crianças e adolescentes é o número de medidas utilizadas. A realização da medida da pressão arterial em apenas um dia parece superestimar os reais valores. Chiolero et al.,37 após avaliarem 6763 crianças em três diferentes dias, encontraram prevalências de PAE de $11,4 \%, 3,8 \%$ e de $2,2 \%$ no primeiro, segundo e terceiro dia, respectivamente. Tais achados são semelhantes aos de Anand et al. 6 que, apesar de aferirem a pressão arterial em uma única visita, mediram por três vezes, encontrando valores de $3,2 \%$ na primeira aferição, $0,8 \%$ na segunda e finalmente $0,46 \%$ na última aferição. Os resultados de Sorof et al.24 corroboram os dos estudos acima, 6,37 pois as prevalências de PAE registradas em seu inquérito foram de $19,4 \%, 9,5 \% \mathrm{e}$ $4,0 \%$ para a primeira, segunda e terceira medida da pressão arterial, respectivamente.

\section{Referências}

1. Yusuf S, Reddy S, Ounpuu S, Anand S. Global burden of cardiovascular diseases: part I - general considerations, the epidemiologic transition, risk factors, and impact of urbanization. Circulation. 2001; 104: 2746-53.

2. Salcedo-Rocha AL, Alba JE, Contreras-Marmolejo M. Classifying Mexican adolescents' high blood pressure, associated factors and importance. Rev Salud Publica (Bogota). 2010; 12: 612-22.

3. Moura AA, Silva MA, Ferraz MR, Rivera IR. Prevalence of high blood pressure in children and adolescents from the city of Maceió, Brazil. J Pediatr (Rio J). 2004; 80: 35-40.

4. Urrutia-Rojas X, Egbuchunam CU, Bae S, Menchaca J, Bayona M, Rivers PA, Singh KP. High blood pressure in school children: prevalence and risk factors. BMC Pediatr. 2006; 16; 6: 32.

5. Christofaro DG, Fernandes RA, Polito MD, Romanzini M, Ronque ER, Gobbo LA, Oliveira AR. A comparison between overweight cutoff points for detection of high
$\mathrm{O}$ único fator metodológico praticamente unânime, entre os estudos componentes desta revisão, foi o critério para classificar os sujeitos com ou sem PAE. Todos os estudos determinaram que valores superiores ao percentil 95 seriam os mais corretos para essa classificação.

Uma limitação da presente revisão é o fato de apenas um dos autores ter realizado a seleção e leitura de todos os artigos, o que pode ter reduzido sua sensibilidade na detecção de estudos de prevalência de PAE ou hipertensão entre crianças e adolescentes. Todavia, os descritores, os critérios de inclusão e exclusão foram estabelecidos por consenso entre os pesquisadores e seguidos de forma criteriosa na continuidade dos trabalhos.

Em resumo, nos estudos avaliados na presente revisão, foram identificadas variações nas metodologias empregadas, especialmente no que refere à categorização da faixa etária, que pode ter contribuído significativamente para a ampla variação das prevalências encontradas. Ressalta-se que o único fator praticamente comum em todos os estudos foi a classificação de PAE dos sujeitos com valores de PA acima do percentil 95. Apesar da ampla variação das prevalências de PAE, observa-se que tais estudos são importantes para auxiliar em políticas e ações de saúde pública, fornecendo indícios para o combate da crescente prevalência de PAE nas faixas etárias mais jovens, contribuindo dessa forma para a prevenção da instalação deste fator de risco cardiovascular na vida adulta. Porém, as comparações entre os diferentes estudos devem ser realizadas com cautela. blood pressure in adolescents. J Pediatr (Rio J). 2009; 85: 353-8.

6. Anand NK, Tandon L.Prevalence of hypertension in school going children. Indian Pediatr. 1996; 33: 377-81.

7. Oliveira AM, Oliveira AC, Almeida MS, Almeida FS, Ferreira JB, Silva CE, Adan LF. Environmental and anthropometric factors associated with childhood arterial hypertension. Arq Bras Endocrinol Metabol. 2004; 48: 849-54.

8. Sánchez-Zamorano LM, Salazar-Martinez E, AnayaOcampo R, Lazcano-Ponce E. Body mass index associated with elevated blood pressure in Mexican school-aged adolescents. Prev Med. 2009; 48: 543-8.

9. Ostrowska-Nawarycz L, Nawarycz T. Prevalence of excessive body weight and high blood pressure in children and adolescents in the city of Łódź. Kardiol Pol. 2007; 65: 1079-87.

10. Monyeki KD, Kemper HC, Makgae PJ. The association of fat patterning with blood pressure in rural South African 
children: the Ellisras Longitudinal Growth and Health Study. Int J Epidemiol. 2006; 35: 114-20.

11. Monego ET, Jardim PC. Determinants of risk of cardiovascular diseases in schoolchildren. Arq Bras Cardiol. 2006; 87: 37-45.

12. Saleh EA, Mahfouz AA, Tayel KY, Naguib MD, Bin-alShaikh NM. Hypertension and its determinants among primary school children in Kuwait: an epidemiological study. East Mediterr Health J. 2000; 6: 333-7.

13. Salvadori M, Sontrop JM, Garg AX, Truong J, Suri RS, Mahmud FH, Macnab JJ, Clark WF. Elevated blood pressure in relation to overweight and obesity among children in a rural Canadian community. Pediatrics. 2008; 122: 8217 .

14. Silva KS, Faria Jr JC. Fatores de risco associados à pressão arterial elevada em adolescentes. Rev Bras Med Esporte. 2007; 13: 237-40.

15. Mavrakanas TA, Konsoula G, Patsonis I, Merkouris BP. Childhood obesity and elevated blood pressure in a rura population of northern Greece. Rural Remote Health. 2009; 9: 1150

16. Borges LM, Peres MA, Horta BL. Prevalence of high blood pressure among schoolchildren in Cuiabá, Midwestern Brazil. Rev Saúde Pública. 2007; 41: 530-8.

17. Genovesi S, Giussani M, Pieruzzi F, Vigorita F, Arcovio C, Cavuto S, Stella A. Results of blood pressure screening in a population of school-aged children in the province of Milan: role of overweight. J Hypertens. 2005; 23: 493-7.

18. McNiece KL, Poffenbarger TS, Turner JL, Franco KD, Sorof JM, Portman RJ. Prevalence of hypertension and prehypertension among adolescents. J Pediatr. 2007; 150: 6404

19. Chiolero A, Madeleine G, Gabriel A, Burnier M, Paccaud F, Bovet P. Prevalence of elevated blood pressure and association with overweight in children of a rapidly developing country. J Hum Hypertens. 2007; 21: 120-7.

20. Chiolero A, Paccaud F, Bovet P. Pre-hypertension and hypertension among adolescents of Switzerland. J Pediatr (Rio J.). 2007; 151: 24-5

21. Jafar TH, Islam M, Poulter N, Hatcher J, Schmid CH, Levey AS, Chaturvedi N. Children in South Asia have higher body mass-adjusted blood pressure levels than white children in the United States: a comparative study. Circulation. 2005; 111: 1291-7.

22. Gomes BM, Alves JG. Prevalence of high blood pressure and associated factors in students from public schools in Greater Metropolitan Recife, Pernambuco State, Brazil, 2006. Cad Saúde Pública. 2009; 25: 375-81

23. Paradis G, Lambert M, O’Loughlin J, Lavallée C, Aubin J, Delvin E, Lévy E, Hanley JA. Blood pressure and adiposity in children and adolescents. Circulation. 2004; 110: 1832-8.

24. Sorof JM, Lai D, Turner J, Poffenbarger T, Portman RJ. Overweight, ethnicity, and the prevalence of hypertension in school-aged children. Pediatrics. 2004; 113: 475-82.
25. Task Force on Blood Pressure Control in Children. Report of the second Task Force on Blood Pressure Control in Children. Pediatrics. 1987; 79: 1-25.

26. Task Force on Blood Pressure Control in Children. Update on the 1987 Task Force Report on High Blood Pressure in Children and Adolescents: a Working Group Report from the National High Blood Pressure Education Program National High Blood Pressure Education Program Working Group on Hypertension Control in Children and Adolescents. Pediatrics. 1996; 98: 649-58.

27. National High Blood Pressure Education Program Working Group on Hypertension Control in Children and Adolescents. The Fourth Report on the diagnosis, evaluation, and treatment of high blood pressure in children and adolescents. Pediatrics. 2004; 114: 555-76.

28. Christofaro DG, Andrade SM, Fernandes RA, Ohara D, Dias DF, Freitas Júnior. Prevalence of risk factors for cardiovascular diseases among students of Londrina, PR: differences between economical classes. Rev Bras Epidemiol. 2011; 14: 27-35.

29. Fernandes RA, Christofaro DG, Buonani C, Araújo LR, Kassab YK, Cardoso JR, Freitas IF Jr, Machado DR. The accuracy of national body fat cutoff levels in the prediction of elevated blood pressure among Brazilian male adolescents. J Trop Pediatr. 2010; 56: 208-9.

30. Fernandes RA, Christofaro DG, Codogno JS, Buonani C, Bueno DR, Oliveira AR, Freitas Júnior IF. Proposal of cut points for the indication of abdominal obesity among adolescents. Arq Bras Cardiol. 2009; 93: 558-63.

31. Park MK, Menard SW, Yuan C. Comparison of auscultatory and oscillometric blood pressures. Arch Pediatr Adolesc Med. 2001; 155: 50-3.

32. Furusawa EA, Ruiz MF, Saito MI, Koch VH. Evaluation of the Omron 705-CP blood pressure measuring device for use in adolescents and young adults. Arq Bras Cardiol. 2005; 84: $367-70$

33. Christofaro DG, Fernandes RA, Gerage AM, Alves MJ, Polito MD, Oliveira AR. Validation of the Omron HEM 742 blood pressure monitoring device in adolescents. Arq Bras Cardiol. 2009; 92: 10-5

34. Kolios K. Arterial hypertension. In: Konstantopoulos A, editor. Clinical Paediatrics. Athens: Iatrikes Ekdoseis Zita; 2006-2007. p. 937-40

35. Biro FM, Daniels SR, Similo SL, Barton BA, Payne GH, Morrison JA. Differential classification of blood pressure by fourth and fifth Korotkoff phases in school-aged girls. The National Heart, Lung and Blood Institute Growth and Health Study. Am J Hypertens. 1996, 9: 242-7.

36. Moraes AC, Fulaz CS, Netto-Oliveira ER, Reichert FF. Prevalence of metabolic syndrome in adolescents: a systematic review. Cad Saúde Pública. 2009; 25: 1195-202.

37. Chiolero A, Cachat F, Burnier M, Paccaud F, Bovet P. Prevalence of hypertension in schoolchildren based on repeated measurements and association with overweight. J Hypertens. 2007; 25: 2209-17.

Recebido em 30 de novembro de 2010

Versão final apresentada em 16 de agosto de 2011

Aprovado em 16 de setembro de 2011 